\title{
GROUPS HAVING AT MOST THREE IRREDUCIBLE CHARACTER DEGREES
}

\author{
I. M. ISAACS
}

In several earlier papers of this author and D. S. Passman, attempts were made to determine as far as possible the structure of finite groups satisfying certain hypotheses made upon the set of degrees of their irreducible complex characters. In particular, in [4], groups with a.c. $m$, that is with all nonlinear irreducible characters having the same degree $m$, were studied. Among the conclusions that were drawn about such groups is that they must be metabelian. In the present paper we prove

THEOREM. Let $G$ be a finite group with at most three irreducible character degrees. Then $G$ is solvable and has derived length $\leqq 3$.

Since the simple groups $\mathrm{SL}\left(2,2^{a}\right)$ for $a \geqq 2$ have character degrees $1,2^{a}-1,2^{a}$ and $2^{a}+1$, there exists no extension of our theorem to the case of four character degrees. The author is not aware, however, of an example of a solvable group whose derived length is greater than its number of irreducible character degrees.

Before proceeding with the proof of the theorem, we state here a part of Proposition 2.2 of [3] which will be needed.

Proposition. Let $E$ be a finite group satisfying $E^{\prime \prime}=1<E^{\prime}$ and $E^{\prime} \subseteq H$ for all $H$ with $1<H \triangle E$. Then either $E$ is a p-group or $E$ is a Frobenius group with cyclic complement and elementary abelian kernel.

Proof of The Theorem. Let $d(G)$ denote the number of distinct irreducible character degrees of $G$. We prove by induction on $|G|$ that if $d(G) \leqq 3$, then the derived length of $G$ is $\leqq d(G)$. This is clear for $d(G)=1$ and has been proved in [4] for $d(G)=2$. We shall not use that result, however, since it follows from the present proof with essentially no extra work.

CASE 1. Suppose there exists a prime $p$ such that $p \mid \chi(1)$ for every nonlinear $\chi \in \operatorname{Irr}(G)$. (This case must occur if $d(G)=2$.) By a result of Thompson, (see Theorem 2.5 (i) of $[4]$ ), $G$ has a normal $p$-complement $H$. Let $\theta \in \operatorname{Irr}(H)$ and let $\chi$ be an irreducible constituent of the induced character $\theta^{G}$. Then $[\chi \mid H, \theta] \neq 0$ and since $G / H$ is supersolvable, there exists a subgroup $K$, with $H \subseteq K \subseteq G$ and an irreducible character $\psi$ of $K$ such that $\psi^{G}=\chi$ and $\psi \mid H=\theta$. Then $\chi(1)$ $=[G: K] \theta(1)$ and since $p \nmid \theta(1)$ and $[G: K]$ is a power of $p, \theta(1)$ is the

Recelved by the editors May 15, 1968. 
$p^{\prime}$-part of $\chi(1)$. It follows that if $G$ has $r$ irreducible character degrees which are powers of $p$ then $d(H) \leqq d(G)-r+1 \leqq d(G)$. By induction, the derived length of $H$ is $\leqq d(G)-r+1$. If $H^{\prime}=1$, then $G$ is an $M$ group by Satz 18.4 on p. 580 of [1] and by Satz 18.6 on p. 581, our result follows in this case. Suppose then that $H^{\prime}>1$. Now, $G / H^{\prime}$ has a normal abelian $p$-complement so by Ito's Theorem, (Satz 17.10 on p. 570 of [1]), every irreducible character of $G / H^{\prime}$ has degree which is a power of $p$ and thus $d\left(G / H^{\prime}\right) \leqq r \leqq 3$ and by induction, $G / H^{\prime}$ has derived length $\leqq r$. Since the length of $H$ is $\leqq d(G)-r+1$, by induction, the length of $H^{\prime}$ is $\leqq d(G)-r$ and we have the derived length of $G$ is $\leqq(d(G)-r)+r=d(G)$.

CASE 2. $G$ has irreducible character degrees $1, m$ and $n$ with $n \neq 1$ $\neq m$ and $(n, m)=1$. Suppose there exists $H$, with $1<H \triangle G$ and $G / H$ nonabelian. Let $K \supseteq H, K \triangle G$ be maximal such that $G / K$ is nonabelian. By the inductive hypothesis, $G / K$ is solvable and it follows that $E=G / K$ satisfies the hypotheses of the Proposition. The irreducible characters of $E$ may be viewed as characters of $G$ and thus $E$ has a nonlinear irreducible character $\phi$, with $\phi(1)=m$ (say). Let $\chi \in \operatorname{Irr}(G)$ with $\chi(1)=n$. If $E$ is a $p$-group, then $p \mid m$ so $p \nmid n$ and since $G / K$ is a $p$-group, it follows that $\chi \mid K$ is irreducible. Viewing $\phi$ $\in \operatorname{Irr}(G)$ it follows that $\phi \chi \in \operatorname{Irr}(G)$, (see for instance Proposition 1.1 of [2]) and since this character has degree $m n$, this is a contradiction. Thus $E$ is a Frobenius group with cyclic complement and elementary abelian $q$-group as kernel. Let $Q / K$ be the kernel. We have $[G: Q]$ $=\phi(1)=m$. Let $\theta \in \operatorname{Irr}(Q)$. We claim that if $\theta(1)>1$, then $\theta(1)=n$ so $d(Q)=2$ and thus $Q^{\prime \prime}=1$. Since $G^{\prime} \subseteq Q$ we will be done.

Suppose then $\theta(1)>1$. For each linear character $\mu$ of $Q / K$ we let $T(\mu)$ be the inertia group of $\mu \theta$ in $G$. If for any $\mu, T(\mu)=Q$ then $(\mu \theta)^{G}$ is irreducible and $(\mu \theta)^{G}(1)=[G: Q] \theta(1)=m \theta(1)$. Since $m \nmid n$ we must have $m \theta(1)=m$ and $\theta(1)=1$, a contradiction. Thus all $T(\mu)>Q$. The number of these groups is $[Q: K]$. If for every $\mu_{1} \neq \mu_{2}$ we have $T\left(\mu_{1}\right) \cap T\left(\mu_{2}\right)=Q$, then $U_{\mu}(T(\mu) / Q-1)$ is disjoint and we have $[Q: K] \leqq[G: Q]$. However, $G / K$ is a Frobenius group so $[G: Q] \mid([Q: K]-1)$. This is a contradiction and it follows that $T\left(\mu_{1}\right)$ $\cap T\left(\mu_{2}\right)>Q$ for some pair $\mu_{1} \neq \mu_{2}$. Choose $g \notin Q$ with $g \in T\left(\mu_{1}\right) \cap T\left(\mu_{2}\right)$. We have

$$
\mu_{i} \theta=\left(\mu_{i} \theta\right)^{0}=\mu_{i}^{0} \theta^{0}
$$

and it follows that

$$
\left(\mu_{1}^{g}\right)^{-1} \mu_{1} \theta=\theta^{g}=\left(\mu_{2}^{g}\right)^{-1} \mu_{2} \theta .
$$

Let $\lambda_{i}=\left(\mu_{i}^{o}\right)^{-1} \mu_{i}$ so $\lambda_{1} \lambda_{2}^{-1} \theta=\theta$. If $\lambda_{1} \lambda_{2}^{-1}=1$, then $\left(\mu_{1} \mu_{2}^{-1}\right)^{g}=\left(\mu_{1} \mu_{2}^{-1}\right)$. 
However, since $G / K$ is a Frobenius group, the only linear character of $Q / K$ which is fixed by any element outside of $Q$ is the principal character and this yields $\mu_{1}=\mu_{2}$, a contradiction. Thus $\lambda_{1} \lambda_{2}{ }^{-1} \neq 1$ and $\theta$ vanishes off the kernel of this character. It follows that $\theta \mid K$ is reducible and thus $q \mid \theta(1)$, and $q \nmid m$. Let $\chi$ be an irreducible constituent of $\theta^{G}$ so $\theta(1) \mid \chi(1)$ and hence $\chi(1) \neq 1$ or $m$. Thus $\chi(1)=n$ and since $n$ is relatively prime to $[G: Q], \chi \mid Q$ is irreducible. However, $\theta$ is a constituent of $\chi \mid Q$ and thus $\theta=\chi \mid Q$ and therefore $\theta(1)=n$, our claim is established and the proof of this case is complete.

CASE 3. The degrees of the irreducible characters of $G$ are $1, m$ and $n$ with $1<m<n$ and $(m, n)=1$. Also, for every $H$ such that $1<H \triangle G$ we have $G / H$ is abelian. The proof of the theorem will be complete if we show that this situation is impossible.

Let $\chi$ be an irreducible nonlinear character of $G$. If $\operatorname{ker} \chi>1$ we would have $G / \operatorname{ker} \chi$ abelian, contradicting the nonlinearity of $\chi$. Thus every nonlinear irreducible character of $G$ is faithful. Suppose now that $\chi(1)=m$. Since $G$ has an irreducible character of degree $n$, it follows from a theorem of Burnside and Brauer (see Satz 10.8 on p. 519 of [1]) that some power $\chi^{r}$ has an irreducible constituent of degree $n$. Choose $r$ minimal with this property and let $\phi \in \operatorname{Irr}(G), \phi(1)$ $=n$ with $\phi$ a constituent of $\chi^{r}$. Then for some irreducible constituent $\psi$ of $\chi^{r-1}$ we must have

$$
0 \neq[\psi \chi, \phi]=\frac{1}{|G|} \sum_{x \in G} \psi(x) \chi(x) \overline{\phi(x)}=[\bar{\psi}, \chi \bar{\phi}]
$$

and by the minimality of $r$ we have $\psi(1)=m$. (The case that $\psi$ is linear is impossible since then $\psi \chi$ is irreducible of degree $m$.) Thus $\chi \Phi$ has an irreducible constituent of degree $m$ and has no linear constituent $\lambda$ since otherwise

$$
0 \neq[\chi \bar{\phi}, \lambda]=[\bar{\phi}, \bar{\chi} \lambda]
$$

contradicting $\bar{\phi}(1)=n>m=\bar{\chi} \lambda(1)$. Thus all irreducible constituents of $\chi \bar{\phi}$ have degree $m$ or $n$ and at least one has degree $m$. Let $a$ be the number of constituents of degree $m$ and $b$ the number of those of degree $n$. We obtain $m n=a m+b n$. Now, $n \mid a m$ and since $(m, n)=1$, we have $n \mid a$. However $a>0$ and thus $a \geqq n$. It follows that $a=n$ and $b=0$ so every irreducible constituent of $\chi \bar{\phi}$ has degree $m$. We may write

$$
\chi \bar{\phi}=\sum_{i=1}^{n} \theta_{i}
$$

where the $\theta_{i} \in \operatorname{Irr}(G)$ all have degree $m$ and are not necessarily all 
distinct. Suppose some $\theta_{i}$ is not of the form $\lambda \chi$ for any linear character $\lambda$. Then for all such $\lambda$, we have $0=\left[\lambda \chi, \theta_{i}\right]=\left[\lambda, \bar{\chi} \theta_{i}\right]$ and $\bar{\chi} \theta_{i}$ has no linear constituents. However $0 \neq\left[\chi \bar{\phi}, \theta_{i}\right]=\left[\bar{\phi}, \bar{\chi} \theta_{i}\right]$ so $\bar{\chi} \theta_{i}$ has a constituent $\Phi$ of degree $n$. Let $c$ be the number of irreducible constituents of $\bar{\chi} \theta_{i}$ of degree $m$ and $d$ the number of degree $n$. Then as above we have $m^{2}=c m+d n$. Thus $m \mid d$ and $d>0$ so $d \geqq m$ and we have $m^{2} \geqq d n$ $\geqq m n$ which contradicts $n>m$. It follows that each $\theta_{i}$ is of the form $\chi \lambda_{i}$ for some linear character $\lambda_{i}$ of $G$. This yields

$$
\chi \Phi=\chi \sum_{i=1}^{n} \lambda_{i}
$$

If $x \in G^{\prime} \subseteq \operatorname{ker} \lambda_{i}$ we obtain $\phi(x) \chi(x)=n \chi(x)$ and since $\Phi$ is faithful, $\Phi(x) \neq n$ for $x \neq 1$. Thus $\chi(x)=0$ for all $x \in G^{\prime}-1$. This yields $\left[\chi \mid G^{\prime}, 1\right]$ $\neq 0$ so $G^{\prime} \subseteq$ ker $\chi$ and this contradiction completes the proof of the theorem.

\section{REFERENCES}

1. B. Huppert, Endliche Gruppen 1, Springer-Verlag, Berlin, 1967.

2. I. M. Isaacs and D. S. Passman, Groups with representations of bounded degree, Canad. J. Math. 16 (1964), 299-309.

3. - A characterization of groups in terms of the degrees of their characters, Pacific J. Math. 15 (1965), 877-903.

4. - A characterization of groups in terms of the degrees of their characters. II, Pacific J. Math. 24 (1968), 467-510.

University of Chicago 